\title{
Uso Racional de Corticoides en el Paciente con Shock Séptico
}

\author{
JORGE ROQUE E. ${ }^{1}$ \\ 1. Médico jefe Unidad Cuidados Intensivos de Pediatría, Clínica Alemana de Santiago de Chile. \\ Profesor de Pediatría, Universidad del Desarrollo, Chile.
}

\begin{abstract}
Rational Use of Corticoids in a Patient with Septic Shock

Introduction: Septic shock (SS) is a significant cause of mortality in NICUs. Objective: Review current knowledge on Hypothalamic-Pituitary-Adrenal Axis (HPA) and the scientific support for the use of glucocorticoids in the use of this clinical picture. We know that The patient's ability to evolve into improvement or worsening depends upon the ability of the HPA axis to develop and sustain an adequate response to the stress provoked by SS. In some patients, due to many reasons, the prolongation of SS leads to a deficit of cortisol that results in functional acute adrenal insufficiency. Cortisol levels do not respond to ACTH stimulation test. There is no consensus among authors as to what is a normal concentration of cortisol during stress, or even if it is correlated with death among children with SS. The American College of Critical Care Medicine guidelines for SS in Pediatrics and Neonatology have made some recommendations for use of hydrocortisone.

(Key words: Septic shock, adrenal insufficiency, ACTH, cortisol.).

Rev Chil Pediatr 2010; 81 (1): 12-19
\end{abstract}

\section{RESUMEN}

Introducción: El shock séptico (SS) es una de las mayores causas de mortalidad en unidades de cuidados intensivos pediátricas. Objetivo: Revisar qué sabemos hasta ahora del papel que juega el eje hipotálamopituitaria-adrenal (HPA) en el SS y si hay evidencia científica que apoye el uso de glucocorticoides en el transcurso de este cuadro. Sabemos que de la habilidad del eje HPA para montar y sostener en el tiempo una adecuada respuesta al stress provocado por un SS dependerá si el paciente evoluciona a la mejoría o se agrava. En algunos pacientes debido a múltiples mecanismos, la prolongación del SS puede llevar a un déficit de cortisol, resultando en una insuficiencia adrenal aguda o funcional, la que se reflejaría en que los niveles de cortisol no responderían con un incremento significativo frente a una prueba de estimulo con ACTH. Entre los diversos reportes revisados, no existe consenso sobre cual sería la concentración "normal" de cortisol durante el stress y más aun no está claro si esto se correlacionaría con la mortalidad en el caso de los niños. Las guías de shock séptico en pediatría y neonatología del American College of Critical Care Medicine recomiendan en que tipo de pacientes con SS se debiera considerar tratamiento con hidrocortisona.

(Palabras clave: Shock séptico, insuficiencia adrenal, ACTH, hidrocortisona).

Rev Chil Pediatr 2010; 81 (1): 12-19

Trabajo recibido el 05 de junio de 2009, devuelto para corregir el 03 de agosto de 2009, segunda versión el 05 de septiembre de 2009, aceptado para publicación el 02 de octubre de 2009.

Correspondencia a:

Dr. Jorge Roque E.

E-mail: jroque@alemana.cl 


\section{Introducción}

Desde que en 1855 Thomas Addison reportó por primera vez el cuadro de insuficiencia de la función de las glándulas suprarrenales (GSR), mucho se ha avanzado en el conocimiento acerca de la fisiopatología de esta enfermedad. La asociación posterior de insuficiencia adrenal (IA) e infección a principios del siglo XX y el rápido avance en la química orgánica permitieron identificar y sintetizar la adrenalina y los esteroides producidos por dicha glándula. A mediados del siglo $\mathrm{XX}$ se pudo comprobar el papel fundamental que tienen las GSR en la respuesta del organismo al stress. En las últimas dos décadas se ha producido evidencia clínica y experimental que relaciona el shock séptico con la IA y la inflamación sistémica inducida por resistencia de los receptores de glucocorticoides, lo que ha llevado a plantear el tratamiento con bajas dosis de hidrocortisona en el curso del shock séptico, tratando una hipotética IA asociada a este cuadro para impactar positivamente en la mortalidad $^{1}$.

El shock séptico es una de las mayores causas de mortalidad en las unidades de cuidados intensivos pediátricas (UCIP) y de adultos ${ }^{2}$. Clásicamente se distinguen la insuficiencia adrenal primaria (déficit de producción de cortisol y ocasionalmente aldosterona por la corteza adrenal causado por una enfermedad congénita o adquirida e la glándula), la secundaria (déficit de producción de ACTH a nivel de la pituitaria anterior) y la terciaria (déficit de producción de CRH en el hipotálamo).

El objetivo del artículo es revisar qué sabemos hasta ahora del papel que juega el eje hipotálamo-pituitaria-adrenal (HPA) en el shock séptico y si hay evidencia científica que apoye el uso de glucocorticoides en el transcurso de este cuadro. Antes recordaremos conceptos básicos.

\section{Anatomía de la GSR}

La GSR esta constituida por 2 zonas independientes, la medula y la corteza. La medula adrenal es considerada parte del sistema nervioso simpático y produce las catecolaminas vasopresoras epinefrina y norepinefrina, las que son almacenadas en la misma médula. La corteza adrenal consta de 3 zonas, la más externa, zona glomerulosa (15\%) de la cual hay una gradual transición a la zona media o fasciculata $(75 \%)$ y de esta una clara transición a la zona más interna en contacto con la médula, zona reticularis (10\%). La zona glomerulosa es el sitio primario de síntesis de mineralocorticoides, la zona fasciculata esta involucrada en la síntesis de glucocorticoides y la zona reticularis predominantemente en la de andróge$\operatorname{nos}^{2,3}$.

\section{Esteroidogénesis}

Las 3 principales clases de hormonas producidas por la corteza adrenal son los glucocorticoides (cortisol), mineralocorticoides (aldosterona) y andrógenos (testosterona, androstenediona, DHEA). Todos los esteroides están compuestos del mismo núcleo esterol presente en el colesterol. Aunque las células esteroidales adrenales pueden sintetizar colesterol, el $80 \%$ del colesterol usado en la esteroidogenesis proviene de lipoproteínas plasmáticas. El cortisol circula en el plasma en una forma libre y activa (5-10\%), y otra inactiva, unida reversiblemente a proteínas (albúmina y cortisolbinding globulin o CGB $)^{2,3}$.

\section{Fisiología del Eje Hipotálamo-Pituitaria- Adrenal (HPA)}

La producción de corticosteroides es regulada por el eje HPA. La producción de cortisol es estimulada por la hormona adrenocorticotrófina (ACTH), la cual es un péptido de 39 aminoácidos secretado en la glándula pituitaria anterior, que se produce a partir de la fractura de una molécula mucho mayor llamada pro-opiomelanocortina y que también libera otros pépticos ( $\beta$-endorfina, lipotropina, hormona estimulante de melanocito). La ACTH se libera en pulsos, logrando un ritmo diurno (circadiano) después de la infancia, con mayor concentración plasmática de ACTH y cortisol temprano en la mañana (6 a 8 AM). La vida media de la ACTH es corta y la elevación del cortisol en respuesta al estímulo ACTH es a los pocos minutos después de su pulso. La ACTH también estimula la síntesis de andrógenos y de manera menos importante la de mineralocorticoides. 
La secreción de ACTH es regulada por la hormona liberadora de corticotrofina $(\mathrm{CRH})$ y también, aunque de manera menos importante por la vasopresina (VA), ambas son secretadas por el hipotálamo. La CRH es sintetizada por las neuronas del núcleo paraventricular y la VA por los núcleos paraventricular y supraoptico, almacenándose esta última en la pituitaria posterior. Otros factores estimulantes de la secreción de ACTH son las catecolaminas, la angiotensina II, el péptido intestinal vasoactivo y algunas citokinas (IL-1, IL-2, IL-6, FNT- $\alpha$ ). A su vez los corticosteroides ejercen una retroalimentación negativa sobre el eje HPA, inhibiendo secreción de ACTH, así como la trascripción del gen de pro-opiomelanocortina, la producción de CRH y VA. También la ACTH inhibe su propia secreción por retroalimentación negativa.

La secreción de aldosterona está regulada principalmente por el sistema renina-angiotensina y la kalemia. Fundamentalmente frente a una disminución del volumen intravascular se produce secreción de renina por el aparato yuxtaglomerular del riñón. La renina es una enzima proteolítica que fracciona una proteína mayor producida en el hígado, el angiotensinógeno, produciéndose angiotensina I la cual es nuevamente fraccionada por la enzima convertidora de angiotensina (producida en pulmón y otros tejidos) a angiotensina II. Si bien esta última será transformada en angiotensina III, ambas tienen potente efecto estimulador de secreción de aldosterona ${ }^{1-4}$.

\section{Acción de los Esteroides Adrenales}

Los glucocorticoides actúan sobre el metabolismo de los carbohidratos, proteínas y lípidos, modulan el tono vascular y modulan la respuesta inmune, entre otros. Aumentan la gluconeogénesis hepática, glicólisis, proteolisis y lipólisis, en algunos tejidos disminuyen la captación celular de glucosa, producen hiperglicemia y estimulan el depósito de glicógeno en el hígado. En el sistema inmune ellos suprimen el proceso inflamatorio. Con altas dosis de glucocorticoides se produce depleción de monocitos, eosinófilos, linfocitos (principalmente T), disminución de la síntesis de in- munoglobulinas, estimulación de la apoptosis de linfocitos y disminución de la síntesis de citokinas proinflamatorias (IL-1, IL-6 y FNT $\alpha$ ). Los glucocorticoides tienen un efecto inótropo positivo sobre el corazón y aumentan la presión sanguínea mediante una serie de mecanismos que involucran al sistema vascular y los riñones. Inducen balance negativo de calcio por aumento de la excreción renal y disminución de la absorción intestinal.

Los niveles básales de cortisol han sido estudiados en sujetos voluntarios sanos, encontrándose un amplio rango de valores, que en promedio es de $15 \mu \mathrm{g} / \mathrm{dL}$ en la mañana, pero bajo condiciones de stress como fiebre o convulsiones se elevaba sobre $18 \mu \mathrm{g} / \mathrm{dl}$. De manera similar al stress, si se les administraba $1 \mathrm{mg}$ de ACTH, el cortisol plasmático aumentaba sobre los $18 \mu \mathrm{g} / \mathrm{dL}$. En cambio en pacientes con IA (ejemplo: hiperplasia suprarrenal congénita) sus niveles básales de cortisol resultaban menores de $4 \mu \mathrm{g} / \mathrm{dL}$ y post estimulación con ACTH menores de $18 \mu \mathrm{g} /$ dl (Ref 1).

La principal función de los mineralocorticoides es mantener el volumen intravascular, lo cual es logrado mediante la retención de sodio y excreción de potasio e hidrogeniones ${ }^{1-4}$.

\section{Efecto del Stress Sobre la Función Adrenal}

Los corticoides son los principales mediadores de la respuesta al stress. De inmediato se produce un aumento de la secreción de ACTH, lo que es seguido de un aumento concomitante de cortisol. Los niveles de CBG disminuyen y consecuentemente los de cortisol libre aumentan, pero si se mide la concentración total de cortisol en el plasma se puede encontrar disminuida debido a la hipoproteinemia concomitante. Se suma un cambio en el número y sensibilidad de los receptores de glucocorticoides tisulares y una pérdida del ciclo circadiano, secundario a un incremento de producción $\mathrm{CRH}$ y ACTH estimuladas por citokinas, acción vagal y reducción en la retroalimentación negativa del cortisol sobre el eje HPA. La IL-1 aumenta por mayor secreción de los macrófagos en respuesta a reacciones inflamatorias e inmunes. Al mismo tiempo el eje HPA incrementa el cortisol plasmático, lo que resulta en 
una retroalimentación negativa sobre los macrófagos. Las mismas citokinas proinflamatorias IL-1, IL-2, IL-6, FNT- $\alpha$ que estimulan la secreción de $\mathrm{CRH}$ son inhibidas posteriormente por el cortisol. Todos estos cambios durante el stress tienen por objeto mantener la homeostasis, es así como por ejemplo la hiperglicemia permite la redistribución de la glucosa hacia los tejidos dependientes de insulina, y el aumento de cortisol apunta a mantener la reactividad cardiovascular normal. En el fondo, de la habilidad del eje HPA para montar y sostener en el tiempo una adecuada respuesta al stress provocado por una sepsis, dependerá en gran parte si el paciente evoluciona a la mejoría o se agrava. Probablemente al inicio del shock se producen incrementos de $\mathrm{CRH}, \mathrm{ACTH}$, y cortisol, que habitualmente son proporcionales al grado de stress que provoca la enfermedad, pero posteriormente debido a múltiples mecanismos, la prolongación de la enfermedad puede llevar a un déficit de cortisol, resultando en una IA aguda. En términos prácticos esto significa que en un paciente con shock séptico, no solo debiera evaluarse su cortisol basal plasmático (debiera estar elevado), sino que también, su capacidad de respuesta frente a un estimulo como la administración de ACTH para ver si tiene "reserva" funcional que le permita continuar manteniendo en el tiempo una respuesta proporcional al stress. Esto introduciría un nuevo concepto de IA, el cual se refiere a aquellos pacientes que teniendo un nivel adecuado de cortisol plasmático frente al stress, no son capaces de mantenerlo en el tiempo y se reflejaría en que no responderían con un incremento significativo de los niveles de cortisol frente a una prueba con ACTH. Se le ha llamado IA relativa y no tiene un sustrato anatómico conocido ${ }^{1-6}$.

\section{Insuficiencia Adrenal (IA) en el Shock Séptico}

La IA ha sido observada en el $30-70 \%$ de los casos de shock séptico en niños y adultos. $\mathrm{Su}$ frecuencia depende de la definición del umbral del nivel cortisol basal plasmático y del incremento del cortisol a la prueba de estimulación con ACTH que se haya usado en cada serie.
El problema es que entre los diversos autores y estudios realizados tanto en adultos como en niños, no existe consenso sobre cual sería la concentración "normal" de cortisol durante el stress y más aun no está claro si esto se correlacionaría con la mortalidad (tabla 1). Se sabe que independiente de la definición que se use, los pacientes que presentan IA tienden a evolucionar con un shock refractario a resucitación con volumen y terapia con catecolaminas. Los estudios clínicos en adultos sugieren que en dichos casos el tratamiento con glucocorticoides mejora el pronóstico. Se han propuesto valores normales de cortisol plasmático durante el stress, cuyos rangos irían entre 10 y $20 \mu \mathrm{g} /$ dL (tabla 2). También se ha sugerido utilizar la prueba de estimulación con ACTH, frente a la cual se debiera producir un aumento neto de cortisol plasmático de $9 \mu \mathrm{g} / \mathrm{dl}$ o más para ser considerado una prueba de función normal. Esta diferencia o delta de cortisol plasmático también es controvertida, ya que hay autores que definen como positiva la respuesta con aumentos de cortisol de $7 \mu \mathrm{g} / \mathrm{dl}$ o más (Tabla 2) $)^{1-11}$.

\section{Diagnóstico de IA en el Shock Séptico}

Ante la necesidad de conocer la prevalencia de IA en niños con shock séptico y siguiendo las experiencias descritas en adultos, los primeros estudios pediátricos usaron dosis de estimulación con ACTH elevadas $(125-250 \mu \mathrm{g})$, pero posteriormente estas fueron consideradas dosis farmacológicas, especialmente en los niños más pequeños. Tales dosis suprafisiológicas pueden pasar por alto la resistencia de la GSR a la ACTH y resultar en una respuesta normal en niños con IA relativa o funcional asociada al shock séptico. Entonces se planteó que dosis más pequeñas de $\mathrm{ACTH}(1 \mu \mathrm{g})$ pueden hacer que esta prueba sea más sensible para detectar la IA relativa. Obviamente este elemento también afectará la prevalencia de IA relativa en niños con shock séptico ${ }^{2,3,5,6,12}$.

\section{Mecanismos de IA en el Shock Séptico}

Varios mecanismos han sido propuestos para explicar la IA asociada a sepsis. Primero, daño anatómico en la GSR, pituitaria o hipotálamo puede causar déficit de producción 
Tabla 1. Estudios reportados de asociación entre mortalidad y función adrenal en el shock séptico en niños

\begin{tabular}{|lll|}
\hline Estudio & Conclusión \\
\hline Hatherill M. Arch Dis Child 1999; 80 (1): 51-5 & $\begin{array}{l}\text { Insuficiencia adrenal es común en niños con SS. Se } \\
\text { asocia con aumento de requerimiento vasopresor y duración del shock. } \\
\text { Mortalidad no fue diferente entre los que presentaron IA y aquellos con } \\
\text { adecuada función adrenal. }\end{array}$ \\
\hline Riordan FA. Crit Care Med 1999; 27: 2257-2261 & $\begin{array}{l}\text { Niveles de cortisol significativamente mas bajos fueron encontrados en los } \\
\text { fallecidos comparados con los sobrevivientes con meningococcemia. }\end{array}$ \\
De Kleijn ED. Pediatr Infect Dis J 2002; 21(4): 330-6 & $\begin{array}{l}\text { Bajo cortisol sérico en combinación con ACTH elevada se asociaron a mayor } \\
\text { mortalidad en niños con meningococcemia grave. }\end{array}$ \\
Pizarro CF. Crit Care Med 2005; 33: 855-859 & $\begin{array}{l}\text { La contribución de la IA a la mortalidad no fue evidente. IA fue frecuente en } \\
\text { niños con SS refractario a catecolaminas y estuvo ausente en niños con SS } \\
\text { que respondió a volumen }\end{array}$ \\
Sarthi M. Pediatr Crit Care Med 2007; 8: 23-28. & $\begin{array}{l}\text { No hubo diferencias de mortalidad entre aquellos pacientes con SS } \\
\text { asociado a IAR y aquellos sin IAR asociada. IAR se asoció a SS refractario a } \\
\text { catecolaminas. }\end{array}$ \\
\hline
\end{tabular}

SS: Shock séptico; IA: insuficiencia adrenal; IAR: insuficiencia adrenal relativa; ACTH: hormona adrenocorticotrófina.

Tabla 2. Definiciones e incidencia de Insuficiencia Adrenal en niños de acuerdo a reportes de diferentes autores publicadas en la literatura internacional

\begin{tabular}{|c|c|c|c|}
\hline \multicolumn{2}{|c|}{ Definición de Insuficiencia Adrenal } & \multicolumn{2}{|c|}{ Incidencia Insuficiencia Adrenal } \\
\hline Autor (año) & Nivel Cortisol ( $\mu \mathrm{g} / \mathrm{dL})$ & Población estudiada & IA (\%) \\
\hline $\begin{array}{l}\text { Hatherill M (1999) } \\
\left({ }^{*} \text { АCTH } 250 \mu \mathrm{g}\right)\end{array}$ & Aumento $<7,5$ post $\mathrm{ACTH}$ & 33 & 52 \\
\hline $\begin{array}{l}\text { Menon K (2002) } \\
\left({ }^{*} \text { ACTH 125-250 } \mu \mathrm{g}\right)\end{array}$ & Cortisol basal $<7 \mathrm{y} / \mathrm{o}<18$ post ACTH & 13 & 31 \\
\hline Pizarro C (2005) & IAA: cortisol basal $<20$ e incremento $\leq 9$ post $\mathrm{ACTH}$ & 57 & 18 \\
\hline$\left({ }^{*} \mathrm{ACTH} 250 \mu \mathrm{g}\right)$ & IAR: cortisol basal $\geq 20$ e incremento $\leq 9$ post ACTH & 57 & 26 \\
\hline Sarthi M (2007) & IAA: cortisol basal $<7$ y pico $<18$ post $A C T H$ & 30 & 0 \\
\hline$\left({ }^{*} \mathrm{ACTH} 1 \mu \mathrm{g}\right)$ & IAR: incremento $<9$ post $A C T H$ & 30 & 30 \\
\hline \multirow[t]{3}{*}{ Hebbar K (2009) } & Cortisol basal $<25$ & 73 & 58 \\
\hline & Cortisol basal $<15$ & 73 & 32 \\
\hline & Cortisol basal $<7$ & 73 & 7 \\
\hline
\end{tabular}

IA: Insuficiencia Adrenal; IAA: Insuficiencia Adrenal Absoluta; IAR: insuficiencia Adrenal Relativa. * Dosis de ACTH usada por cada autor.

de cortisol. Se ha descrito una incidencia de $7-10 \%$ de isquemia o hemorragia en cualquiera de estos componentes del eje HPA en el transcurso de un shock séptico. Esto se podría explicar por un aumento en el flujo arterial hacia estas estructuras, concomitante con un drenaje venoso disminuido. También es posible que apoptosis neuronal se pueda gatillar por la misma inflamación hipotalámica y so- bre-expresión a óxido nítrico sintetasa. Otras sustancias como la sustancia $\mathrm{P}$, radicales súperoxido, monóxido de carbono y prostaglandinas se pueden acumular en el hipotálamo o la pituitaria alterando la síntesis y pulsatilidad de hormonas. Segundo y muy importante para los médicos que trabajamos en UCIP, la síntesis de cortisol puede verse iatrogénicamente afectada por el uso de drogas que alteren la función 
de las enzimas que interviene en la síntesis de cortisol. Varias se han descrito disminuyendo la síntesis de cortisol, como el etomidato, ketoconazol, fluconazol o fenitoina. Una simple dosis de etomidato para intubar a un paciente, induce IA hasta por 48 horas, aumentando el riesgo de muerte. Tercero, el transporte de cortisol unido a CBG y albúmina puede verse alterado por la disminución en los niveles de estas dos proteínas en las etapas más precoces del shock séptico. Es cierto que esto puede aumentar la fracción libre de cortisol en plasma, pero la CBG es muy importante en la entrega de cortisol en los tejidos más inflamados. Las enzimas elastasas producidas por los neutrófilos polimorfonucleares activados en el sitio de la infección pueden específicamente fraccionar la unión de cortisol a CBG, permitiendo al cortisol allí liberado entrar directamente en los tejidos inflamados. O sea, una baja de CBG inducida por un shock séptico puede disminuir la liberación de cortisol en los sitios más inflamados, exacerbando la resistencia de los tejidos al cortisol. Cuarto, puede haber una disminución en el número y actividad de los receptores de glucocorticoides, alterando la capacidad de las células de responder adecuadamente al cortisol $^{1-4}$.

\section{Diagnóstico Clínico}

El cuadro clínico de IA durante el curso de un shock séptico es inespecífico. Los síntomas pueden ser fiebre, dolor abdominal, hipotensión arterial refractaria en el tiempo, vómitos y alteración del estado de conciencia. Otros indicadores pueden ser la refractariedad del shock o inestabilidad cardiovascular a la terapia con volumen generoso o a las catecolaminas. No hay tiempo suficiente para observar la hiperpigmentación de la piel en la clásica IA. Los hallazgos de laboratorio pueden ser como en el cuadro típico de IA: hiponatremia con exceso de sodio urinario, hiperkalemia e hipoglicemia. Se describe eosinofilia, que raramente es hallada ${ }^{1-4}$.

Respecto de las pruebas de laboratorio específicas, en la UCIP, en el escenario de un shock séptico, con un paciente grave, no hay mucho tiempo para decidir, y como se mencionó anteriormente, no hay un consenso en los niveles de cortisol plasmático considerados diagnósticos, sino un amplio rango de valores sugeridos por los diversos autores tanto en adultos como niños. Sin embargo, una prueba dinámica que permite diagnosticar IA relativa es el test de ACTH.

Los criterios de laboratorio diagnóticos de acuerdo a Aneja y Carcillo, utilizando la prueba de la Metapyrona, en pacientes pediátricos son los siguientes: a) IA absoluta: nivel pico de cortisol plasmático post ACTH menor de $18 \mathrm{mg} / \mathrm{dL}$; b) IA relativa o funcional: nivel basal de cortisol plasmático mayor de $18 \mu \mathrm{g} / \mathrm{dL}$ e incremento de cortisol post ACTH menor de $9 \mathrm{mg} / \mathrm{dL}$ y c) Disfunción de la pituitaria: nivel de cortisol basal menor de $5 \mu \mathrm{g} / \mathrm{dL}$ y nivel pico de cortisol mayor a $18 \mu \mathrm{g} / \mathrm{dL}$. En una disfunción central, con déficit de producción de ACTH, se puede realizar la prueba de la metapyrona, que mide la habilidad del eje HPA para responder a una reducción aguda de los niveles de cortisol plasmático. La metapyrona inhibe la enzima 11-hidroxilasa involucrada en el último paso de la síntesis de cortisol. Su inhibición causa una disminución de cortisol y consecuentemente en un aumento compensatorio de la ACTH y del precursor del cortisol, el 11-deoxicortisol. La administración de metapyrona $(30 \mathrm{mg} / \mathrm{kg}$, dosis máxima 3000 $\mathrm{mg}$ ) se hace a medianoche, tomando niveles de cortisol y 11-deoxicortisol plasmáticos al día siguiente a las 8 AM. La respuesta normal debiera ser una disminución de cortisol a menos de $5 \mu \mathrm{g} / \mathrm{dL}$ y un aumento de 11-deoxicortisol a más de $7 \mu \mathrm{g} / \mathrm{dL}$. Esta prueba se debe hacer con pacientes que tengan vía enteral disponible y muy cautelosamente ya que puede causar una IA aguda.

En cuanto a la prueba de estimulación adrenal con ACTH, esta se puede hacer con una dosis de 1,125 o $250 \mu \mathrm{g}$, administrada intravenosa o intramuscular, midiendo los niveles de cortisol antes de la ACTH (basal), a los 30 y 60 minutos después (post ACTH). Por ahora, se sugiere usar dosis de $1 \mu \mathrm{g}$ en niños ${ }^{1-4}$.

\section{Tratamiento del la IA en el Shock Séptico}

En el caso de un paciente con shock séptico asociado a una IA absoluta, como podría ser el caso de una enfermedad de Addison, la 
recomendación es tratarla con hidrocortisona, en dosis de $2-50 \mathrm{mg} / \mathrm{kg}$ en un bolo intravenoso y posteriormente seguir con una infusión continua de $2-50 \mathrm{mg} / \mathrm{kg}$ en 24 horas. Como la hidrocortisona tiene una vida media de 8-12 horas, también se puede administrar intermitentemente. La amplia dosis de 2 a $50 \mathrm{mg} / \mathrm{kg}$, debe ser titulada de acuerdo a la respuesta terapéutica que se observe a nivel cardiovascular. En el caso de los pacientes con IA relativa, las dudas persisten en cuanto al beneficio que podría representar el tratar estos pacientes con hidrocortisona. Hasta ahora los estudios no han demostrado categóricamente que los pacientes pediátricos con IA asociada a shock tengan mayor mortalidad (tabla 1), incluso una gran revisión de Markovitz de la base de datos del Pediatric Health Information System (PHIS) analizando el uso de esteroides en niños entre 0 y 17 años con diagnóstico de sepsis encontró que había un aumento de la mortalidad asociada al uso de corticoides $^{13}$. Menon, en una encuesta que realizó entre intensivistas y endocrinólogos pediátricos encontró que el $81 \%$ de los intensivistas creía que la IA se presentaba a menudo en pacientes críticamente enfermos, mientras que el $41 \%$ de los endocrinólogos creía que nunca o muy raramente se presentaba. En este mismo estudio el $51 \%$ de los intensivistas reconoció que trataba empíricamente con esteroides a los pacientes con hipotensión arterial, mientras que el $81 \%$ de los endocrinólogos refería que nunca o muy ocasionalmente recomendaría tratar empíricamente a un paciente con este fundamento ${ }^{14}$.

Esta claro que se necesitan más estudios que aclaren si realmente el tratamiento con esteroides beneficia a los niños que cursan con sepsis asociada a IA relativa. Sobre estos argumentos, parece prudente no recomendar el uso de corticoides por ahora en este tipo de pacientes, hasta que más estudios aclaren las dudas actuales.

Los pacientes que presentan una falla de la pituitaria con déficit de producción de ACTH endógena, pero que tienen un aumento de cortisol plasmático con la ACTH exógena, debieran tratarse con hidrocortisona de la misma manera que un paciente con IA absoluta. El déficit de producción de ACTH se esta obser- vando cada vez con más frecuencia, ya que el uso rutinario de corticoides en los niños ha ido aumentando, en la medida que más especialistas los incluyen en sus terapias. Niños con cáncer, trasplantados, asmáticos, con laringitis obstructivas, alergias y enfermedades autoinmunes reciben diferentes esquemas de esteroides por tiempos variables. Rix $\mathrm{M}^{15}$, reportó que un tratamiento acotado con corticoides puede suprimir el eje HPA entre 6 semanas y 6 meses. Entonces, aunque no existen estudios que avalen la presente recomendación, frente a un paciente con antecedentes de uso de corticoides y en el contexto de un shock séptico grave con mala respuesta a resucitación con volumen y a catecolaminas, parece razonable el uso de hidrocortisona ${ }^{1}$. Finalmente, el año 2009 las últimas guías de shock séptico en pediatría y neonatología del American College of Critical Care Medicine (ACCCM) hace la siguiente recomendación: "Si un niño está en riesgo de insuficiencia adrenal absoluta o fracaso del eje HPA, (ejemplo: púrpura fulminans, hiperplasia suprarrenal congénita, uso de esteroides reciente, anomalías hipotálamopituitarias) y permanece en shock a pesar de infusión continua de epinefrina o noradrenalina, se le puede administrar hidrocortisona, idealmente después de obtener una muestra de sangre para posterior determinación de la concentración de cortisol plasmático. La hidrocortisona puede ser administrada intermitentemente o en infusión continua en dosis de $1-2 \mathrm{mg} / \mathrm{kg} /$ dia, para estrés hasta $50 \mathrm{mg} / \mathrm{kg} /$ dia para reversión del shock." ${ }^{16}$.

En resumen la IA relativa o funcional en niños es un problema clínico desafiante que requiere más estudios para su definición y manejo. No esta claro en niños que la IA asociada a sepsis aumente la mortalidad. El stress provocado por la sepsis afecta la función del eje HPA y aumenta el nivel de cortisol plasmático. El cuadro clínico de IA es similar al de la sepsis o el shock en los niños. IA iatrogénica secundaria a drogas o tratamientos con glucocorticoides es cada vez más frecuente en niños. Por ahora el ACCCM recomienda tratar con hidrocortisona pacientes con shock séptico asociado a IA absoluta o falla del eje HPA y shock refractario a catecolaminas. 


\section{Referencias}

1.- Aneja R, Carcillo JA: What is the rationale for hydrocortisone treatment in children with infection-related adrenal insufficiency and septic shock? Arch Dis Chil 2007; 92: 165-9.

2.- Maxime $V$, Lesur $O$, Annane D: Adrenal Insufficiency in septic shock. Clin Chest Med 2009; 30: 17-27.

3.- Polito A, Aboab J, Annane D: Adrenal Insufficiency in sepsis. Revista Brasileira Terapia Intensiva 2006; 18: 86-94.

4.- Hassoun A, Sharon Oberfield S: Sdrenal Dysfuntion. En Rogers' Textbook of Pediatric Intensive care. Fourth Edition, 2008 by Lippincott Williams \& Wilkins, a Wolters Kluwer business, Philadelphia, PA, USA. Páginas 1584-98.

5.- Pizarro CF, Troster EJ, Damiani D, Carcillo JA: Absolute and relative adrenal insufficiency in children with septic shock. Crit Care Med 2005; 33: 855-9.

6.- Sarthi M, Lodha R, Vivekanandhan S, Arora NK: Adrenal status in children with septic shock using low-dose stimulation test. Pediatr Crit Care Med 2007; 8: 23-8.

7.- Hatherill M: Adrenal insufficiency in septic shock. Arch Dis Child 1999; 80: 51-5.

8.- Riordan FA, Thompson AP, Ratcliffe JM, Sills JA, Diver $M J$, Hart CA: Admission cortisol and adrenocorticotrophic hormone levels in children with maningococcal disease: Evidence of adrenal insufficiency? Crit Care Med 1999; 27: 2257-61.

9.- De Kleijn ED: Low serum cortisol in combination with high adrenocorticotrophic hormone are associated with poor outcome in chiledren with severe meningococcal disease. Pediatr Infect Dis 2002; 21: 330-6.

10.- Menon K, Clarson C: Adrenal function in pediatric critical illness. Pediatr Crit Care 2002; 3: 112-6.

11.- Hebbar K, Rigby M, Felner E, Easaley K, Fortenberry $J$ : Neuroendocrine dysfunction in pediatric critical illnes. Crit Care Med 2009; 10: 35-40.

12.- Siraux V, De Backer D, Yalavatti G, Melot C, Gervy $C$, Mockel $J$, et al: Relative adrenal insufficiency in patients with septic shock: Comparison of low-dose and conventional corticotropin tests. Crit Care Med 2005; 33: $2479-86$.

13.- Markovitz B, Goodman D, Watson R, Bertoch D, Zimmerman J: A retorspective cohort study of prognostic factors associated with outcome in pediatric severe sepsis: What is the role of steroids?. Pediatr Crit Care Med 2005; 6: 270-4.

14.- Menon K, Lawson M. Identification of adrenal insufficiency in pediatric critical illness. Pediatr Crit care 2007; 8: 276-8.

15.- Rix M, Birkebaek NH, Rosthoj S, Clausen N: Clinical Impact of corticosteroid-induced adrenal suppression during treatment for acute lymphoblastic leukemia in children: a prospective observational study using the low-dose adrenocorticotropin test. J Pediatr 2005; 147 : 645-50.

16.- Brierley J, Carcillo JA, Choong K, Cornell T, DeCaen $A$, Deyman A, et al: Clinical practice parameters for hemodynamic support of pediatric and neonatal septic shock: 2007 update from the American College of Critical Care Medicine. Crit Care Med 2009; 37: 666-88. 